\title{
The Management of Social Media in Major Public Health Emergencies
}

\author{
$\mathrm{Xin} \mathrm{LI}^{1, *}$ \\ ${ }^{1}$ School of Journalism and Communication, Minjiang University, Fuzhou, Fujian 350108
}

\begin{abstract}
In this study, the concept, basic theory and application of the management of social media in major public health emergencies were expounded with the management of social media in the novel coronavirus pneumonia as the breakthrough point. With the related reports or information of the novel coronavirus pneumonia as the analysis sample, the role of social media in the pandemic was discussed from multiple aspects and perspectives by using the method of content analysis. On this basis, the management strategies of social media in major public health emergencies were further proposed.
\end{abstract}

\section{Introduction}

After the outbreak of the novel coronavirus pneumonia in 2020 , social media has become the most important channel for information dissemination. In the long time of home rest, people mainly understood the situation of the pandemic timely through social media, so as to learn the basic knowledge of how to prevent and control the pandemic. However, we also found that there was false information spread by social media during this pandemic period, which had caused unnecessary social panic. This pandemic has certain practical significance for the authorities responsible for the management of media to improve the norms of managing social media.

\section{An Outline of the Management of Social Media}

\subsection{The Concept of Social Media}

Social Media refer to the platforms for the production and exchange of contents based on the relationship of users on the internet. It serves as tools and platforms for people to share opinions, views, experiences and ideas with each other, currently mainly including social networking sites, microblogs, WeChat, blogs, forums, podcasts and so on. Traditional media, such as newspapers, magazines and TV news, are all in one-way spreading and lack instant interaction. The emergence of social media has realized the two-way interaction of media. Users can not only make real-time dialogues, browse information and request ondemand programs, but also participate in discussions, express emotions and express some views on events. Each terminal user can also upload his or her own articles or video data through mobile phones or computers, share them with others for enjoyment, and arouse discussion or resonance. At the same time, social media can also leave complete digital records, not only recording the contents uploaded by the users, but also recording the comments of different users, while making real-time statistics of the account's browsing, the praising and the forwarding of data. As a unique feature of social media, it enriches people's spiritual life and enhances communication between people.

\subsection{The Forms of Social Media}

The most common forms of social media in China are microblogs, WeChat and accounts of other platforms. Taking microblogs as an example, the number of microblog users in China has reached more than 700 million in recent years. Both celebrities and ordinary people can open their own micro-blog accounts, as well as browse, comment and forward the information released by the platform through mobile phones or computers.

\subsection{The Application of Social Media in Major Public Health Emergencies}

As a crisis event, the main process of major public health emergencies can be divided into three stages: crisis prevention, crisis management and crisis recovery. Social media play their roles throughout all these three stages, by the dissemination of information, planning and training for crisis contingency, solving problems and decision-making in a collaborative way and information collection (see Figure 1). 


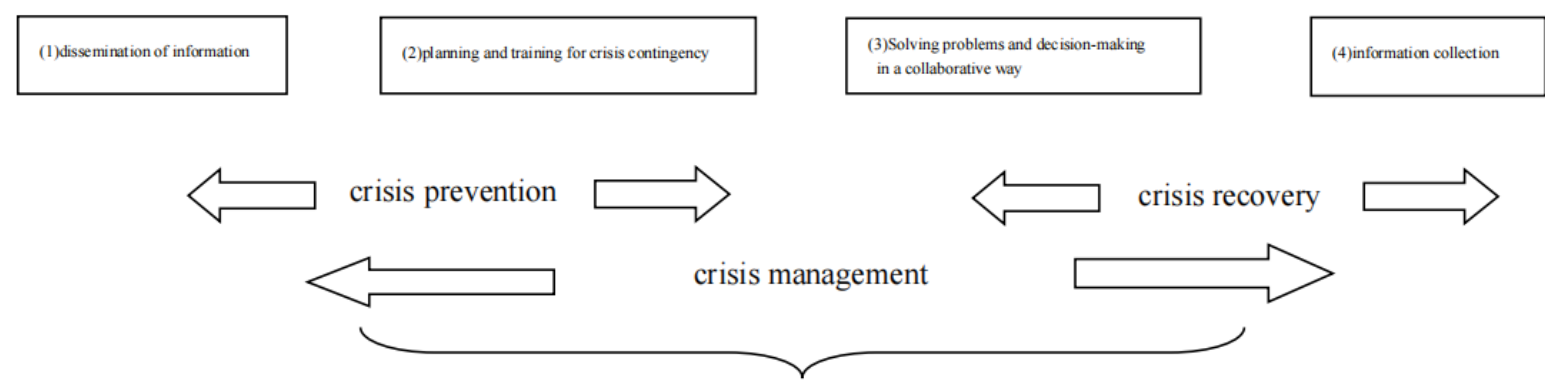

main processes for the management of emergencies

Fig 1. Functions of Social Media in Crisis Events of Major Pandemic

\subsection{Key-Point Information for Social Media to Disseminate in Major Public Health Emergencies}

In major public health emergencies, social media must shoulder corresponding social responsibilities and need to pay attention to the following issues in the dissemination of information:

First, attention should be paid to screening target audiences, such as distinguishing the general public, volunteers, non-governmental organizations and government organizations.

Second, the appropriate way to release information in the process of risk communication should be selected, for example, when reporting the epidemic, a more authoritative platform should be chosen if possible.

Third, the information which the receivers are concerned with or interested in should be released.

Fourth, the negative results caused by error information should be identified, and corresponding plans should be made for the adverse situation in time.

Fifth, information on the risk communication of the infectious diseases should be published in a timely manner and timely feedbacks should be given in response to people's opinions, expert channels should be opened, more experts should be invited to exchange information with the public, and more persuasive forms of contents, such as pictures and videos, should be used to report the pandemic.

Sixth, in reporting the pandemic events of major infectious diseases, the following contents must be included in the reports: What has happened until now? Who is responsible for the incident; whether the victims get the help they deserve; if they get help, how about their situation now; is the crisis effectively controlled or not? What is the next plan? How should the public deal with it? What is the cause of the crisis event?

\section{The Empirical Study of Social Media on the Spread of Novel Coronavirus Pneumonia}

\subsection{Research Methods}

In this study, the technology of Internet search was used, the "novel coronavirus" was taken as the keyword, and the study period was from December 31, 2019 to February 11, 2020. The software of data crawler, Octoparse, was used to search the whole networks, the full texts and the whole media, and a total of 466,241 related first onset news reports were collected. In order to better study the samples, the mode of random sampling was adopted to all the materials so as to obtain the samples needed for the study. According to the statistical requirements for the accuracy of the study, the collected samples were further corrected, and the sample size was finally determined as 2,254.

\subsection{Research Contents}

The modes of presentation, the released contents and the source structures of social media in the spread of the novel coronavirus pneumonia were studied.

\subsection{Results and Analyses}

\subsubsection{The Modes of Presentation of Social Media}

First, publishing channels:

According to the survey, the media which initially released the reports of the novel coronavirus pneumonia were dominated by traditional media, while the initially releasing proportion of other mass portal websites, including social media, was only $24 \%$. There was a huge gap between traditional media and social media in their initially releasing proportions. On the one hand, it is due to the limitations of relevant policies. On the other hand, it reflects that traditional media reports are relatively more authoritative and reliable. The network media is equivalent to the role of the processor in the reporting of the pandemic situation, mainly reprinting the reports of the traditional media, and then further processing the information, such as summarizing the relevant reports and producing features, so as to provide a more convenient platform for the public to fully understand the pandemic information. 


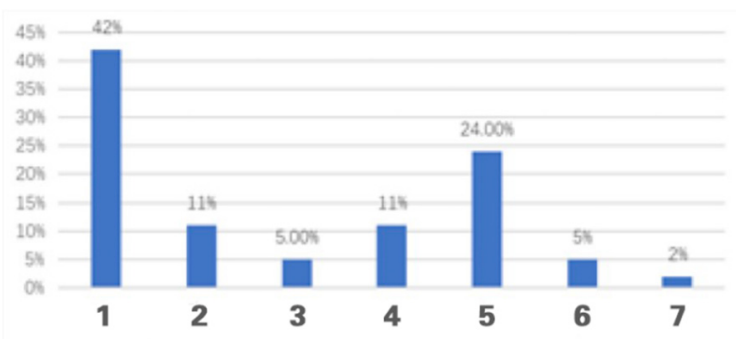

Fig 2. Type structures of the media for first onset reports 1 Central media

2 Local and urban traditional media

3 Financial media

4 Traditional media of science and technology and health

5 Public portal websites

6 Websites of science and technology and health

7 Others

Second, types of news information:

The news reports related to "the novel coronavirus" were divided into "the news-oriented type" and "the knowledge-oriented type". It can be seen that in general, the reports on "novel coronavirus" were mostly "newsoriented", accounting for $79.2 \%$. Therefore, it was regarded that the media reported this pandemic of pneumonia as a social event.

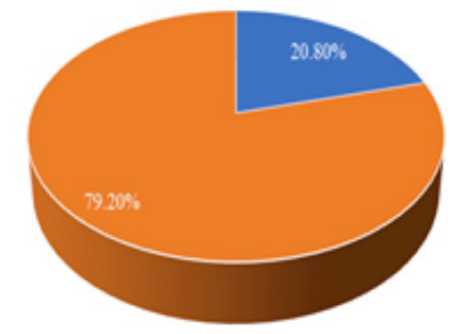

- The news-oriented type. The knowledge-oriented type

Fig 3. The analysis of the structure of the reports by social media of the novel coronavirus pneumonia

\subsubsection{The Released Contents of Social Media}

It can be seen from the analysis results in Fig. 4 that there was no huge difference between the proportions of the related reports about facts and the ones about values of the novel coronavirus pneumonia. The reports about facts (including the knowledge of diseases, the pandemic situation, the government's prevention and control, the introduction to patients, the publicity of features, and the economic impacts and so on) accounted for $50.9 \%$, and the reports about values (including the attitudes to patients, the public psychology, the evaluation of prevention and control, the health education, and the social culture and so on) accounted for $49.1 \%$. Information of facts was necessary for people to understand the situation of the novel coronavirus pneumonia, and this pandemic, as a major public health emergency, also demanded comforts for public psychologies as an essential part. It is also an important function of social media to help the public face the pandemic more rationally and avoid social panic. Judging from the result of responding to this pandemic, the major social media has obviously raised more attention to releasing the information of social psychological value and the information of facts, compared with the news reports of the H1N1 influenza in 2002.

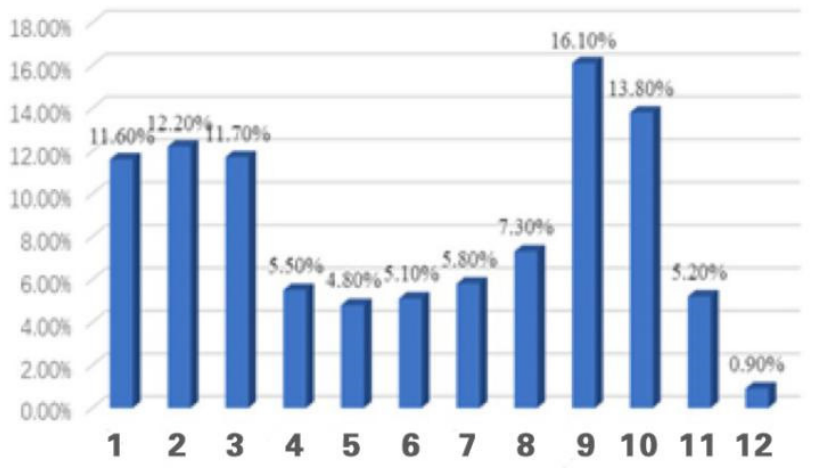

Fig 4. The content structure of the topics for the discussion of reports by social media of the novel coronavirus pneumonia

1 knowledge of diseases

2 pandemic situation

3 governmental prevention and control

4 introduction to patients

5 publicity of features

6 economic impacts

7 attitudes to patients

8 public psychology

9 evaluation of prevention and control

10 health education

11 social culture

12 others

\subsubsection{The Source Structures of the Released Contents by Social Media}

The source results released by social media in this pandemic are shown in Figure 5. The first sources (30.2\%) were "the local health government and the related departments", followed by "the central health system and the related departments", accounting for $15.5 \%$. In addition, medical staff and experts generally covered a reference proportion of $12.2 \%$. It can be seen that the government played a leading role in the release of pandemic-related information, and the status of opinion leaders was also highlighted. For example, academician Zhong Nanshan, academician Li Lanjuan and other authoritative experts have played a great role in helping the public understand the pandemic. 


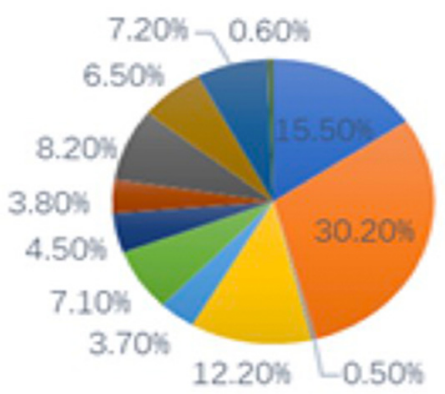
=1
$=2$
$=3$
. 4
= 5
- 6

$=7$

$=8$

$=9$

= 10

= 11

- 12

Fig 5. Source structure of the reports of the novel coronavirus pneumonia

1 Central government system and national health organizations

2 Regional government system and regional health organizations

3 Civil health organizations

4 Medical staff and medical experts

5 Patients and specific population

6 General public

7 Public opinion leaders

8 Journalists and commentators

9 Drug control enterprises

10 World Health Organization

11 Governments of other nations

12 Others

\section{Management Strategies of Social Media in Major Public Health Emergencies}

The openness of the platforms of social media has greatly promoted the formation, dissemination and diffusion of information. Users can express their emotions and opinions through social media and participate in the discussion of social and political life and news events. However, social media, which carries a variety of mixed information of different nature, can also have a negative impact on users' lives. Some users may spread false information in a partial and arbitrary manner due to their limited cognition or extreme and irrational thinking modes, while others may freely publish undocumented information online for entertainment or joking, only to trigger social panic. For example, during the pandemic situation of the novel coronavirus pneumonia, some users released the unconfirmed message that "Shuanghuanglian can prevent the novel coronavirus" on social media, which led to people's rushing to buy Shuanghuanglian overnight and had a great impact on social order. While timely releasing real-time information, the mainstream media and the official should also pay attention to these false rumors and refute them in time. Therefore, the management and guidance of social media in major public health emergencies and the improvement of their social responsibility are very important for the guiding of public opinions and the maintaining of public interests and public order.

\subsection{Timely Construction of Authoritative Information Channels}

Since the response to major public health emergencies often involves professional knowledge, and most of the relevant disposals are also completed by professionals, the public can only obtain information through some media in society about the development background, the development trend and the changes of the pandemic. In this situation, it is crucial to build an authoritative and smooth information channel. Only through the publication of relevant information by authorities and professionals to ensure the timely and transparent dissemination of information can we help dispel people's doubts and avoid the panic or social disorder caused by speculation or misinformation. In the early days of the novel coronavirus pneumonia, there was the rumor spread online that supermarkets were about to be closed while people were facing "the dilemma of food and vegetable supply disruption", resulting in many people's rushing to supermarkets crazily for foods and articles for daily use. After a short period of information confusion, the spread of information about the pandemic has gradually become orderly. The most concerned thing was that the National Health and Family Planning Commission held a daily news conference to announce the situation of pandemic infection, such as the confirmed cases, the suspected cases, the number of cured people and the number of deaths, and introduce the current trend of the national pandemic. At the same time, from the provinces to the cities to the counties, the local authorities also timely informed the society of the local pandemic situation through networks, television broadcasting and other media, and introduced the measures and methods of pandemic prevention and control as well as related medical and health knowledge. The release of a large number of authoritative information timely met the "information hunger" of the masses, stabilized the moods of the people and maintained social order. On this basis, the whole society consciously cooperated with relevant government departments to lay the foundation for effective prevention and control of the pandemic.

\subsection{Focusing on the Monitoring and Early Warning of Public Opinions by Social Media}

Social media provide a convenient channel for people to pay attention to the information related to public health emergencies, and they often express their views on events and their needs through social media at the first time. The early warning of public opinions by social media is to evaluate and analyze the current public opinions of social media, to predict its development trend, and to timely respond to future events. In response to public health emergencies, on the one hand, government departments 
should deal with the incident itself in a timely manner; on the other hand, they need to closely monitor public opinions, especially the monitoring and early warning of the social media represented by microblogs, and understand the public's emotions, attitudes, opinions and appeals about the incident. Only in this way can they solve doubts, respond to queries, and absorb the opinions of the masses, which is also helpful to the solution of the incident Major public health emergencies and related public opinions are associated and intertwined. Only by establishing an integrated mode of collaboration and linkage of "monitoring-early warning-respondingunblocking-releasing-feedbacking" and successively completing the unblocking of public opinions while dealing with public health events can major public health emergencies be resolved.

\subsection{Using Social Media to Actively Guide Public Opinions}

The public opinions reflected by social media are different from the ones reflected by traditional media in their transmission carriers, reflecting the characteristics of the rapid dissemination, the wide influence, the emotionalization and the extremism. In the event of major public health emergencies, the impacts of social media on public opinions are more obvious due to the public's strong attention to events and the widespread application of social media. In order to guide public opinions and stabilize public sentiments, we must seize the characteristics of social media, such as diverse means of communication, strong interaction and multiple users, and make good use of this important communication carrier. On the one hand, after major public health emergencies, authoritative voices should be disseminated through social media in a timely manner. For example, government departments and mainstream media should timely release authoritative information, disseminate the knowledge of prevention and control, and tell anti-pandemic stories through their platform accounts. Social media should use the breadth of network communication and the "reliability" of interpersonal communication to quickly spread correct information and positive energy, and to create a positive and united anti-pandemic atmosphere of public opinions. On the other hand, opinion leaders' influence and communication force in social media should be given full play. Opinion leaders have a large number of fans in social media, and they can play a strong guiding role through their words and deeds to influence their fans' emotions, opinions and behaviors. In major public emergencies, organizing and guiding opinion leaders to participate in information dissemination, refuting rumors, poverty alleviation, resource mobilization, volunteer services, etc. can not only play a good effect of demonstration and guidance, but also help the government departments communicate and interact with the public for public opinions.

The novel coronavirus pneumonia has a great impact on our production and life, but it also catalyzes the function of information dissemination of social media, and provides a large number of cases and research data for the industry and academia. Through the analysis of these cases and data, it can provide decision-making references for the management departments of social media, so that it can better play a positive role in dealing with major public health emergencies.

\section{References}

1. Luo XL. Health information analysis and health promotion in social media. Chinese Journal of Medical Library and Information Science. 2017 Oct 15; 1671-3982: 26-33.

2. Han J, Xiao RL, Hu Y, Tang T, Fang LN. An analysis of the characteristics of the information dissemination mode in online social networks. Journal of Computer Applications. 2013 Jan 1; 1001-9081: 105-107.

3. Pan HF, Zhao CN, Ye DQ. Research progress in information epidemiology. Chinese Journal of Disease Control \& Prevention. 2019 May 10; 16743679: 497-500.

4. Editorial department. A glimpse of the foreign management system of social media. China Information Security. 2014 Jul 15; 1674-7844: 94-96.

5. Wu SJ, Chen B, Chen Sh, Wang L, Zeng X. Effects of new social media management on the efficacy and compliance of children with asthma. Hainan Medical Journal. 2017 Sep 10; 1003-6350.

6. Da JY. American social media's management measures and their characteristics. Research on Transmission Competence. 2017 May 10; 2096-3866: 44.

7. Zhang ZA, Hu ShR. Risks of social media communication and its management strategies: a case study of "self-filming in the operating room". News and Writing. 2015 Mar 5; 1002-2295: 52-55. 